\title{
EGFR signaling in the brain is necessary for olfactory learning in Drosophila larvae
}

\author{
Tasja Rahn, Matthias Leippe, Thomas Roeder, ${ }^{1}$ and Henning Fedders ${ }^{1}$ \\ Zoological Institute, Zoophysiology, University of Kiel, 24098 Kiel, Germany
}

\begin{abstract}
Signaling via the epidermal growth factor receptor (EGFR) pathway has emerged as one of the key mechanisms in the development of the central nervous system in Drosophila melanogaster. By contrast, little is known about the functions of EGFR signaling in the differentiated larval brain. Here, promoter-reporter lines of EGFR and its most prominent activating ligands, Spitz, Keren, and Vein, were used to identify the brain structures relevant for the EGFR pathway. Unexpectedly, promoter activity of all these pathway components was found in the mushroom bodies, which are known to be a higher brain center required for olfactory learning. We investigated the role of the EGFR pathway in this process by using different mutant larvae with reduced pan-neuronal EGFR signaling and those with reduced EGFR signaling in mushroom bodies only. Expression of a dominant-negative form of EGFR as well as silencing of the ligands via RNA interference was applied and resulted in significantly impaired olfactory learning performances. General defects in the ability to taste or smell as well as impaired EGFR signaling during embryonic development could be excluded as major reasons for this learning phenotype. In addition, targeted expression of a constitutively active form of the ligand Spitz also led to a significantly reduced learning ability. Thus, very low levels as well as very high levels of EGFR signaling are deleterious for olfactory learning and memory formation. We hypothesize that EGFR signaling in a certain range maintains a homeostatic situation in the mushroom bodies that is necessary for proper learning and memory.
\end{abstract}

[Supplemental material is available for this article.]

Epidermal growth factor receptor (EGFR) signaling is highly conserved throughout the animal kingdom (Stein and Staros 2006; Sibilia et al. 2007; Fraguas et al. 2011). As one of the major signaling pathways involved in tumor growth and metastasis, it has been extensively studied in mammalian cells and numerous animal models (Arteaga 2006; Hambardzumyan et al. 2011; Mimeault and Batra 2011; Read 2011). Excessive EGFR signaling has been causally linked to the development of various cancers (Moasser 2007; Harris and McCormick 2010; Hatanpaa et al. 2010). By contrast, reduced signaling in the brain has recently been associated with cognitive impairment in Alzheimer's and Parkinson's disease (Chen-Plotkin et al. 2011; Hochstrasser et al. 2012).

Drosophila is well suited to study the molecular basis of human neurological diseases (Bonner and Boulianne 2011; Whitworth 2011) because complex genetic manipulations can be performed to analyze the functions of neurons and neuronally expressed genes in vivo (Venken et al. 2011). Moreover, compared to the situation in mammals, the molecular repertoire of phylogenetically conserved signaling pathways, such as the EGFR pathway, is much simpler in Drosophila (Shilo 2003). At the same time, the nervous systems of mammals and Drosophila share several remarkable similarities, which is particularly true for the olfactory system (Davis 2004; Sato and Touhara 2009). Thus, olfactory classical conditioning assays are most commonly used in Drosophila larvae and adult flies to quantify learning and memory abilities (Selcho et al. 2009; Akalal et al. 2010). According to classical conditioning, different odors are presented in these assays as conditional stimuli that must be associated with aversive

'Corresponding authors

E-mail hfedders@zoologie.uni-kiel.de

E-mail troeder@zoologie.uni-kiel.de

Article is online at http://www.learnmem.org/cgi/doi/10.1101/Im.029934.112. (punishment) or appetitive (reward) stimuli, which are, in turn, defined as unconditional stimuli (Pavlov 1927).

The mushroom bodies (MBs), a specialized region of the insect brain, are essential to associate conditional and unconditional stimuli and therefore are considered the memory centers in adult flies and larvae (Sato and Touhara 2009; Busto et al. 2010; Pauls et al. 2010). Within MB neurons, the adenylyl cyclase (AC) rutabaga is assumed to function as a coincidence detector capable of associating information from both stimuli. This enzyme can be activated synergistically by odor-induced $\mathrm{Ca}^{2+}$ and by tasteinduced $G$ protein-coupled receptor signaling (Gervasi et al. 2010). Subsequent cAMP/PKA pathway activation plays a crucial role in synaptic plasticity and memory storage (Blum et al. 2009; Tomchik and Davis 2009).

However, the possible role of the EGFR pathway in memory function has not yet been investigated. To test whether EGFRmediated signaling is physiologically important in the brain, we first generated promoter-gfp (green fluorescent protein) reporter lines for several pathway components. Unexpectedly, microscopic analyses revealed predominant expression of EGFR and its ligands Spitz, Keren, and Vein in the larval MBs. Subsequently, we used an inducible dominant-negative strategy and RNAi-mediated silencing to specifically impair EGFR pathway activation in the central nervous system (CNS) during larval stages. By employing these animals in olfactory classical conditioning assays, we demonstrated that EGFR signaling is essential for olfactory learning and memory in Drosophila larvae.

\section{Results}

EGFR signaling components are expressed in larval MBs To analyze the expression of EGFR pathway components, we generated promoter-gal4 lines for EGFR and its most prominent 
ligands Spitz, Keren, and Vein. These strains were crossed with a UAS-mCD8::GFP (membrane-bound GFP) line, and immunohistochemistry was performed on whole-mount L3 larval and adult brains of the $F_{1}$ generation. GFP expression was detected using anti-GFP immunohistochemistry. In the adult CNS, no promoter activity of egfr was observed. Of the ligand genes, only spitz and keren showed promoter activity in adult MBs (data not shown). However, in the brain lobes of the larval nervous system, we found distinct promoter activity of the egfr gene, which was restricted to the MBs (Fig. 1A). Moreover, the reporter lines for the three ligand genes spitz, keren, and vein (Fig. 1B-D) also showed promoter activity in the larval brain lobes. Here, again the MBs exhibited the strongest GFP signals (Fig. 1B-D). This observation suggests that EGFR-mediated signaling is of high physiological importance in the larval MBs. As these structures are known as centers for olfactory learning and memory, we performed olfactory learning experiments with larvae with impaired neuronal EGFR signaling.

\section{Impaired olfactory learning in larvae constitutively expressing a dominant-negative EGFR}

Olfactory learning ability was investigated in larvae characterized by pan-neuronal EGFR signaling manipulation. Using panneuronal driver lines ensures that all brain structures relevant for memory formation are targeted. The effect of constitutive and panneuronal expression of a dominant-negative isoform of EGFR (nsyb-gal4 > UAS-EGFR ${ }^{\mathrm{DN}}$ ) compared to that of matching controls (nsyb-gal4 $>\mathrm{w}^{1118}$ ) was analyzed in the olfactory learning en mass assay (Neuser et al. 2005). Control larvae demonstrated a significantly higher learning index compared to those expressing dominant-negative EGFR (Fig. $2 \mathrm{~A}, P=0.0022, n_{\text {learning indices }}=6$, $\left.n_{\text {larvae }}>170\right)$. The median learning index of the experimental
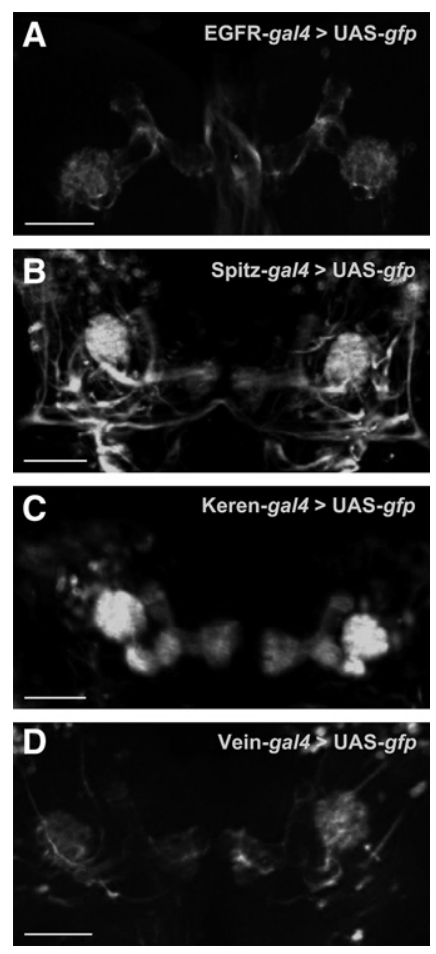

Figure 1. EGFR pathway components are expressed in larval mushroom bodies. Immunostaining of brains of EGFR-gal4 > UAS-gfp larva $(A)$, Spitz-gal4 > UAS-gfp larva (B), Keren-gal4 > UAS-gfp larva (C), and Vein-gal4 > UAS-gfp larva $(D)$. $(A-D)$ Anti-GFP staining indicates expression of the corresponding EGFR pathway genes. Bar, $50 \mu \mathrm{m}$.
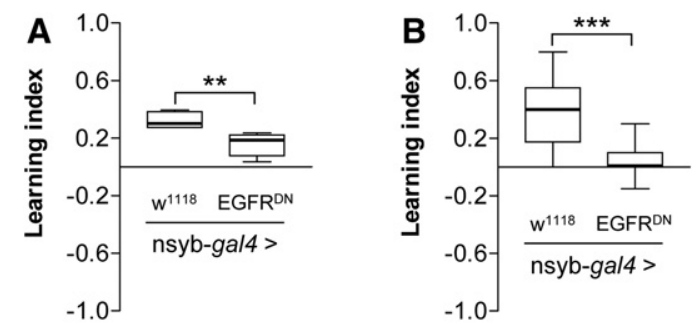

C

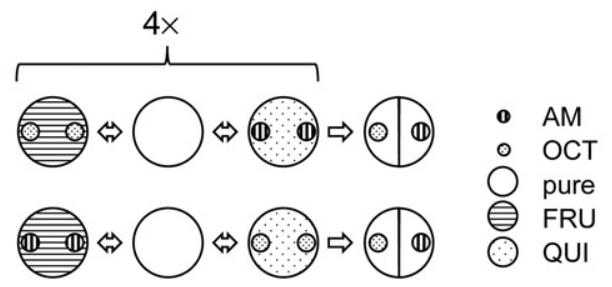

Figure 2. Constitutive and pan-neuronal $E G F R^{D N}$ expression impairs larval learning. $(A, B) F_{1}$ third instar control larvae (nsyb-gal4 $>w^{1118}$ ) were compared with those with constitutively impaired pan-neuronal EGFR signaling (nsyb-gal4 $>$ UAS-EGFR ${ }^{\mathrm{DN}}$ ). Larvae were tested in an en mass assay ( $n_{\text {learning indices }}=6$ each; $n_{\text {larvae }}>170$ each) $(A)$ and in an individual-animal assay ( $n_{\text {learning indices }}=64$ each) (B). (C) Scheme of the olfactory learning assays. In the en mass assay, groups of about 15 larvae were trained and tested together. After $3 \mathrm{~min}$ in an odor-choice situation between $\mathrm{AM}$ and $\mathrm{OCT}$, the number of larvae on the different odor sides was counted. In the individual-animal assay, a group of eight larvae was trained together but tested individually to exclude nonindependent behavior. (AM) Amyl acetate, (FRU) fructose, (OCT) 1-octanol, (QUI) quinine, $\left({ }^{* *}\right) P<0.01,\left({ }^{* * *}\right) P<0.001$.

group (0.2) was reduced to $67 \%$ of that of the control group (0.3). To exclude nonindependent confounding interactions between individuals, larvae were also tested individually using an individual-animal version of the learning assay (Scherer et al. 2003). As shown in en mass assays, control larvae had a significantly better learning capability than those expressing dominant-negative EGFR (Fig. $2 \mathrm{~B}, P<0.0001, n_{\text {learning indices }}=$ 64 each). The median learning index of the control larvae, 0.4 , clearly indicated olfactory learning, whereas the median learning index of larvae expressing EGFR ${ }^{\mathrm{DN}}$ was reduced to 0 .

\section{Constitutive and pan-neuronal expression of EGFR ${ }^{\mathrm{DN}}$ does not impair taste or smell}

Olfactory learning deficits may result from general defects in the ability to taste or smell. To exclude this possibility, naive, untrained larvae were tested for their response to gustatory stimuli and to an olfactory attractant. The gustatory capability of control larvae and of larvae constitutively and pan-neuronally expressing $\mathrm{EGFR}^{\mathrm{DN}}$ was investigated by offering fructose (FRU, attractive taste) or quinine (QUI, repellent taste) compared to pure agarose. Both control larvae and those constitutively and pan-neuronally expressing $\mathrm{EGFR}^{\mathrm{DN}}$ demonstrated significant preferences for FRU over pure agarose (Fig. 3A) and for pure agarose over QUI (Fig. $3 \mathrm{~B})$. Notably, no differences in preferences were detected between these groups of larvae (Fig. 3A, FRU vs. pure, $P=0.6450, n_{\text {Pref }} \geq$ 20; Fig. 3B, QUI vs. pure, $P=0.1026, n_{\text {Pref }} \geq 19$ ).

To test the olfactory abilities of control larvae and larvae constitutively and pan-neuronally expressing $\mathrm{EGFR}^{\mathrm{DN}}$, an odor (amyl acetate $[\mathrm{AM}]$ or 1-octanol [OCT]) versus no-odor setup was used. Both groups demonstrated significant attraction to both odors (Fig. 3C,D). Importantly, the two genotypes did not reveal any significant difference regarding preferences for the odors 

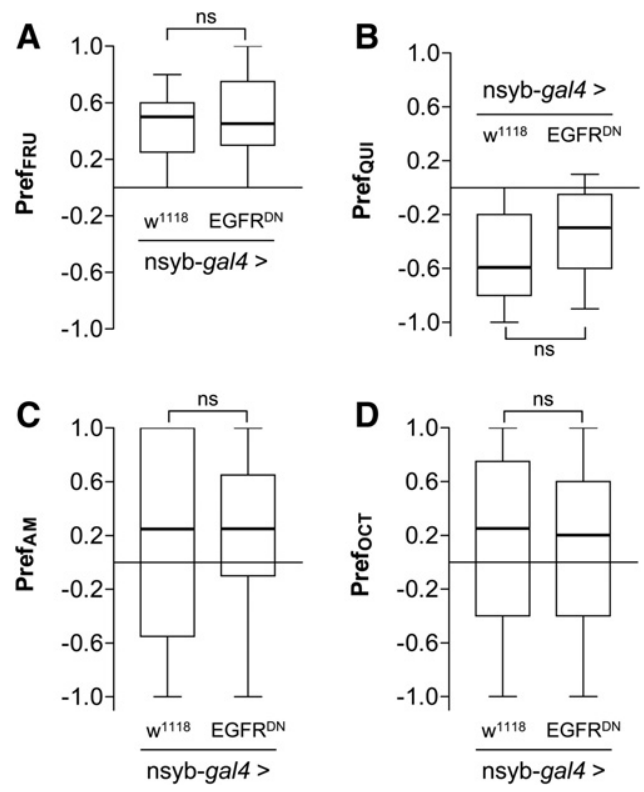

Figure 3. Constitutive and pan-neuronal EGFR ${ }^{\mathrm{DN}}$ expression does not impair taste or smell. $(A, B)$ Gustatory preference (Pref). Control larvae (nsyb-gal4 $>\mathrm{w}^{1118}$ ) and those constitutively and pan-neuronally expressing $\mathrm{EGFR}^{\mathrm{DN}}$ (nsyb-gal4 $>$ UAS-EGFR ${ }^{\mathrm{DN}}$ ) showed the same appetitive response to FRU ( $n_{\text {Pref }} \geq 20$ each) $(A)$ and aversive response to QUI $\left(n_{\text {Pref }} \geq 19\right)(B)$. $(C, D)$ Odor sensing. Control larvae (nsyb-gal4 $>\mathrm{w}^{1118}$ ) and those constitutively and pan-neuronally expressing EGFR ${ }^{\mathrm{DN}}$ (nsyb-gal4 > UAS-EGFR ${ }^{\mathrm{DN}}$ ) did not demonstrate any difference in their preference to the odor of AM ( $n_{\text {Pref }}=50$ each) or OCT $\left(n_{\text {Pref }}=50\right.$ each). (AM) Amyl acetate, (FRU) fructose, (ns) not significant ( $P>$ 0.05), (OCT) 1-octanol, (QUI) quinine.

(Fig. 3C, AM vs. no odor, $P=0.8821$; Fig. 3D, OCT vs. no odor, $P=$ $0.5978 ; n_{\text {Pref }}=50$ each). Thus, these results indicate that larvae constitutively and pan-neuronally expressing $\mathrm{EGFR}^{\mathrm{DN}}$ are impaired in neither taste nor smell. Accordingly, the decreased learning ability of larvae constitutively and pan-neuronally expressing $\mathrm{EGFR}^{\mathrm{DN}}$ can be attributed to a genuine learning defect.

\section{Pan-neuronal induction of EGFR ${ }^{\mathrm{DN}}$ during larval life impairs olfactory learning}

To exclude developmental effects, we used the mifepristoneinducible pan-neuronal elav-GeneSwitch line to reduce EGFR signaling exclusively during larval life (Osterwalder et al. 2001). The elav-GeneSwitch line allows us to manipulate gene expression in a time-controlled and temperature-independent manner, making it advantageous for learning experiments with larvae. Control larvae and those treated with mifepristone during larval life were tested in the individual-animal assay. Notably, induced expression of $\mathrm{EGFR}^{\mathrm{DN}}$ for just $24 \mathrm{~h}$ in late larval stages led to significantly reduced olfactory learning ability (Fig. 4A, $P<0.0001$, $n_{\text {learning indices }}=64$ each). The control group clearly showed olfactory learning, with a median learning index of 0.4 , whereas that of the treated larvae was reduced to 0 . Also, this demonstrates that the insertion of the EGFR ${ }^{\mathrm{DN}}$ transgene does not affect learning ability. To investigate whether mifepristone itself has an impact on memory formation, elav-GS $>\mathrm{w}^{1118}$ control larvae were fed with normal fly food or fly food supplemented with mifepristone for $24 \mathrm{~h}$. Importantly, drug feeding also did not influence the larvae's capability to learn (Fig. $4 \mathrm{~B}, P=0.2737, n_{\text {learning indices }}=64$ ).

In addition, we analyzed the morphology of larval neuropils after induction of $\mathrm{EGFR}^{\mathrm{DN}}$ for $24 \mathrm{~h}$. Brains of larvae constitutively and pan-neuronally expressing EGFR ${ }^{\mathrm{DN}}$ did not show an altered neuropil morphology when compared to brains of uninduced control larvae (Supplemental Fig. 1). Thus, we assume that reduced olfactory learning capabilities in larvae with impaired panneuronal EGFR signaling do not result from disturbances in brain development.

\section{Single EGFR ligands are necessary for olfactory learning}

Because not only promoter activity of the egfr gene but also that of the activating ligand genes was detected in MBs of the larval brain, we investigated the influence of these upstream components on olfactory learning. For this purpose, the individual-animal assay was performed with larvae expressing pan-neuronal RNAi constructs targeting spitz, keren, and vein during larval stages. In contrast to the induction of EGFR ${ }^{\mathrm{DN}}$, which was performed for only $24 \mathrm{~h}$, the expression of ligand ${ }^{\mathrm{RNAi}}$ began immediately after hatching to achieve an adequate silencing effect until experiments were performed with third instar larvae. Again, elavGS $>\mathrm{w}^{1118}$ larvae were used in parallel as a control to exclude that drug feeding alone influences learning ability. Comparing the uninduced controls and the induced ligand ${ }^{\mathrm{RNAi}}$ animals in the individual-animal assay revealed a significant difference in olfactory learning. RNAi directed against each of the three ligand transcripts led to impaired memory formation (Fig. 5A, spitz ${ }^{\mathrm{RNAi}}, P<0.0001$; keren $^{\mathrm{RNAi}}, \quad P<0.0001 ; v_{\text {ein }}{ }^{\mathrm{RNAi}}, P<0.0001 ; n_{\text {learning }}$ indices $=$ 64 each). The uninduced control larvae of all three ligand ${ }^{\mathrm{RNAi}}$ experiments had a median learning index of 0.4 and thus were clearly capable of olfactory learning. The induced ligand ${ }^{\mathrm{RNAi}}$ larvae spitz $^{\mathrm{RNAi}}$, keren $^{\mathrm{RNAi}}$, or vein ${ }^{\mathrm{RNAi}}$ ) were apparently affected in their ability to learn, exhibiting a median learning index of 0 . However, control larvae (elav-GS $>\mathrm{w}^{1118}$ ) that were feeding on fly food supplemented with or without mifepristone did not show any difference in their ability to learn (Fig. 5B, $P=0.6455, n_{\text {learning }}$ indices $=64$ each). Moreover, the odor sensing as well as the gustatory test was applied to all ligand ${ }^{\mathrm{RNAi}}$ larvae to exclude defects in their ability to smell or taste. Uninduced and induced larvae showed the same response to FRU (spitz ${ }^{\mathrm{RNAi}}, P=0.8280$; keren $^{\mathrm{RNAi}}, P=0.9351 ;$ vein $^{\mathrm{RNAi}}, P=0.1469 ; n_{\mathrm{Pref}}=20$ each) or QUI spitz $^{\mathrm{RNAi}}, P=0.7656$; keren $^{\mathrm{RNAi}}, P=0.8071$; vein $^{\mathrm{RNAi}}, P=$ $0.7551 ; n_{\text {Pref }}=20$ each) compared to pure agarose and to AM spitz $^{\mathrm{RNAi}}, \quad P=0.4939 ;$ keren $^{\mathrm{RNAi}}, \quad P=0.2768 ;$ vein $^{\mathrm{RNAi}}, \quad P=$ $0.8601 ; n_{\mathrm{Pref}}=50$ each) or OCT spitz $^{\mathrm{RNAi}}, P=0.3040 ;$ keren $^{\mathrm{RNAi}}$, $P=0.4988 ; v^{2}{ }^{\mathrm{RNAi}}, P=0.7746 ; n_{\text {Pref }}=50$ each) compared to no odor (Supplemental Figs. 2, 3). These experiments clearly
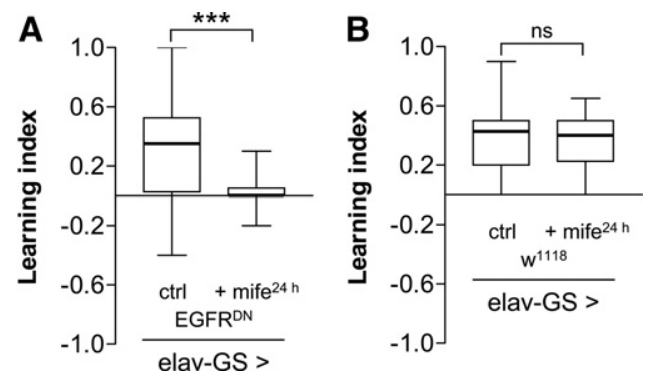

Figure 4. Induction of pan-neuronal $E G F R^{D N}$ for $24 \mathrm{~h}$ reduces olfactory learning. (A) After pan-neuronal EGFR ${ }^{\mathrm{DN}}$ expression for $24 \mathrm{~h}$, olfactory learning ability was significantly impaired in the individual-animal assay ( $n_{\text {learning indices }}=64$ each). (B) An influence of mifepristone on olfactory learning was excluded by feeding normal fly food (ctrl) or fly food supplemented with mifepristone (mife) to elav-GS $>\mathrm{w}^{1118}$ control larvae $\left(n_{\text {learning }}\right.$ indices $=64$ each). (ctrl) Control, (GS) GeneSwitch, (mife) mifepristone, (ns) not significant $(P>0.05),\left({ }^{* * *}\right) P<0.001$. 
A
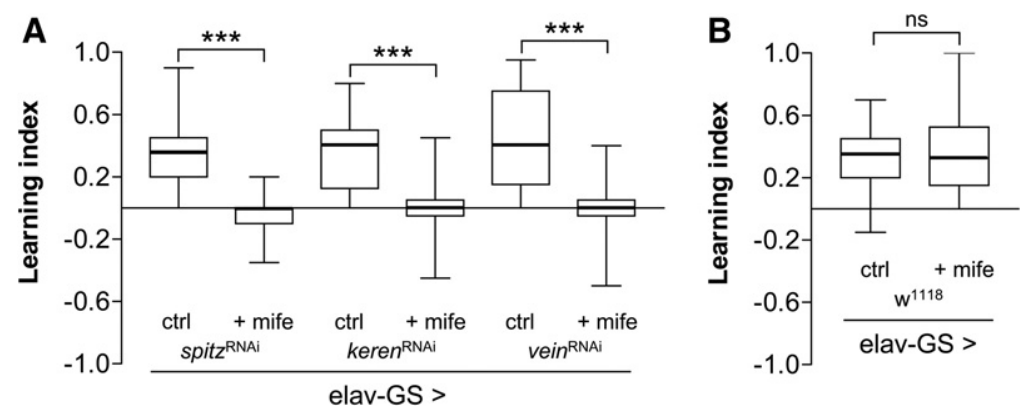

Figure 5. Induction of pan-neuronal RNAi-mediated silencing of EGFR ligands after hatching leads to decreased olfactory learning ability. $(A)$ In the individual-animal assay, olfactory learning capability was decreased in larvae after induction of pan-neuronal RNAi-mediated gene silencing immediately after hatching $\left(n_{\text {learning indices }}=64\right.$ each). $(B)$ Control larvae (elav-GS $>\mathrm{w}^{1118}$ ) were fed with normal fly food (ctrl) or fly food supplemented with mifepristone (mife) to exclude an influence of the agent on olfactory learning ( $n_{\text {learning indices }}=64$ each). (ctrl) Control, (GS) GeneSwitch, (mife) mifepristone, (ns) not significant $(P>0.05),{ }^{(* *)} P<0.001$.

demonstrate that silencing of a single EGF receptor ligand during post-embryonic stages is sufficient to reduce the learning ability of Drosophila larvae.

EGFR signaling balance is important for olfactory learning Next, we assessed whether memory formation is modified by enhanced EGFR signaling. To address this question, we used the individual-animal assay to investigate larvae that pan-neuronally expressed secreted Spitz (sSpitz) induced for $24 \mathrm{~h}$. These larvae exhibited a clearly reduced olfactory learning ability compared to uninduced controls (Fig. $6, P<0.0001, n_{\text {learning indices }}=64$ each). The median learning index of controls was unchanged compared to that of former experiments (0.4), whereas the sSpitz-overexpressing larvae showed only minor learning, with a median learning index of 0.1. Furthermore, sSpitz-overexpressing larvae were not impaired in either taste or smell when applied to the gustatory test (FRU vs. pure, $P=0.6776$; QUI vs. pure, $P=0.8284 ; n_{\text {Pref }}=20$ each) or odor sensing (AM vs. no odor, $P=0.9945$; OCT vs. no odor, $P=0.4855 ; n_{\text {Pref }}=50$ each), respectively (Supplemental Figs. 4, 5).

\section{Olfactory learning is critically dependent on EGFR signaling in the MBs}

The promoter activity of EGFR and its ligands Spitz, Keren, and Vein in the larval MBs was the starting point for the investigation of the role of EGFR signaling in olfactory learning. To analyze the relevance of EGFR signaling exclusively in the MBs, we used the driver line OK107-gal4, which drives expression predominantly to the MBs, to manipulate the pathway and performed the individual-animal learning assay. The effect of constitutive expression of the dominant-negative isoform of EGFR in the MBs (OK107-gal4 > UAS-EGFR ${ }^{\mathrm{DN}}$ ) on olfactory learning behavior was investigated in comparison to that of matching controls $\left(\mathrm{OK} 107\right.$-gal4 $\left.>\mathrm{w}^{1118}\right)$.

Control larvae had a significantly better learning capability than those expressing EGFR ${ }^{\mathrm{DN}}$ exclusively in MBs (Fig. 7, $P<$ $0.0001, n_{\text {learning indices }}=64$ each). The median learning index of the control larvae, 0.5 , clearly indicated olfactory learning, whereas the median learning index of larvae expressing EGFR ${ }^{\mathrm{DN}}$ in the MBs was reduced to 0 . Notably, the negative effect on learning was comparable with that observed in larvae with pan-neuronal $\mathrm{EGFR}^{\mathrm{DN}}$ expression.

\section{Discussion}

To understand the physiological significance of EGFR signaling in the CNS, we elucidated the expression patterns of EGFR pathway components within the Drosophila larval and adult brain using promoter-gfp reporter lines. We found that promoter activity of egfr and its activating ligands spitz, keren, and vein was most apparent in the larval MBs. In particular, no promoter activity of egfr was detected in the adult CNS. Thus, we assumed that EGFR signaling is of high physiological relevance in the differentiated larval brain and, here, is involved in the major task the MBs are known for, learning and memory.

Constitutive and pan-neuronal expression of a dominant-negative form of EGFR strongly reduced olfactory learning in larvae, whereas their ability to taste and smell was not impaired, indicating that the observed learning phenotype depends on central rather than sensory defects. Moreover, using the inducible, mifepristonedependent GeneSwitch system, we demonstrated that this learning phenotype results from impaired EGFR signaling in the functional brain and not from developmental defects caused by impaired signaling during embryogenesis. Interestingly, silencing of each of the EGFR ligand genes spitz, keren, and vein at larval stages as well as enhanced expression of secreted (activated) Spitz led to the same dramatic decrease of olfactory learning ability.

Strikingly, reduction of EGFR signaling exclusively in the MBs revealed the same strong learning defect as was observed in larvae with pan-neuronal manipulations of the pathway. This result is in accordance with the prominent promoter activity of EGFR pathway components in the larval MBs and shows that EGFR signaling within the Kenyon cells of the MBs is of critical importance for proper learning abilities.

The most important result of this study is the finding that proper EGFR signaling is essential for olfactory learning in Drosophila, particularly with regard to our sparse knowledge of EGFR signaling in the developed brain. In vertebrates, EGFR signaling plays multiple roles during embryogenesis and nervous system development, but has also been linked to myelination (Brinkmann et al. 2008; Luo et al. 2011), Alzheimer's disease (Fleck et al. 2011; Woo et al. 2011), schizophrenia (Harrison and Law 2006), and multiple sclerosis (Aguirre et al. 2007). As in

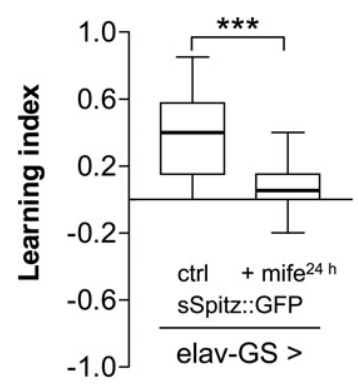

Figure 6. Pan-neuronal activation of EGFR signaling via secreted Spitz (sSpitz) significantly impairs olfactory learning ability. In the individualanimal assay, olfactory learning capability was significantly decreased in those larvae expressing sSpitz for $24 \mathrm{~h}$ compared to the uninduced control larvae ( $n_{\text {learning indices }}=64$ each). (ctrl) Control, (GS) GeneSwitch, (mife) mifepristone, $(* * *) P<0.001$. 


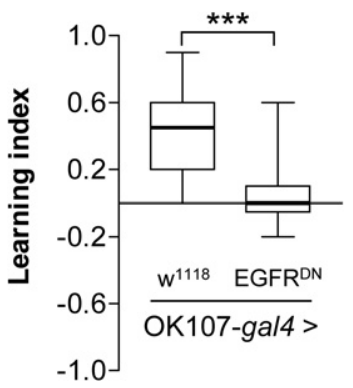

Figure 7. Constitutive induction of $E \mathrm{FR}^{\mathrm{DN}}$ in the larval mushroom bodies reduces olfactory learning. In the individual-animal assay, olfactory learning capability was impaired in those larvae constitutively expressing $\mathrm{EGFR}^{\mathrm{DN}}$ in the larval MBs (OK107-gal4 $>\mathrm{EGFR}^{\mathrm{DN}}$ ) compared to control larvae $\left(\right.$ OK107-gal4 $\left.>\mathrm{w}^{1118}\right)$ ( $n_{\text {learning indices }}=64$ each). $\left.{ }^{* * *}\right) P<0.001$.

vertebrates, little is known about EGFR signaling in the Drosophila nervous system. The EGFR pathway has been identified as important for the regulation of sleep (Foltenyi et al. 2007) as well as for ethanol sensitivity (Corl et al. 2009).

A link between EGFR and signaling systems associated with learning was also recently established. Notably, Hannan et al. (2006) connected EGFR signaling to a novel adenylyl cyclase (AC) pathway in the Drosophila brain. In their experiments, this neurofibromin 1 (NF1)/Ras-dependent pathway was stimulated by growth factors binding to EGFR to activate an as yet unidentified AC. Furthermore, the pathway is assumed to constitute an alternative to the rutabaga AC-mediated pathway in the brain, with both leading to cAMP/PKA signaling, which is in turn a major component associated with short-term memory formation (Blum et al. 2009). These findings imply that EGFR signaling acts upstream of cAMP/PKA and thus regulates olfactory learning via an alternative AC. Moreover, attenuated as well as enhanced PKA signaling has been reported to reduce learning ability in flies (Drain et al. 1991), which is consistent with our observation that the correct balance of EGFR signaling in the brain is important for memory formation.

Why all three EGFR ligands are essential for proper olfactory learning is not yet understood. One possible explanation is that signaling via all ligands is necessary to maintain the proper signaling balance essential for olfactory learning. Alternatively, the system may not be organized in such a manner that different ligands can be functionally exchanged.

Based on the expression patterns observed for all ligands and EGFR, different modes of signaling are possible. During Drosophila brain development, axonal structures are thought to release Spitz to activate the EGFR pathway in glial cells to promote cell survival (Bergmann et al. 2002). Another EGFR ligand, Vein, is produced by pioneer neurons and is required for the survival of longitudinal glia (Hidalgo et al. 2001). Our observations further suggest an alternative signaling system. Because all components of the EGFR signaling system are present in the MBs, autocrine or paracrine modes of signaling are possible. Autocrine EGFR signaling is relevant to various systems ranging from epithelial mechanotransduction (Kojic et al. 2010) to brain tumor development (Tang et al. 1997). Thus, proper autocrine or paracrine EGFR signaling within the Kenyon cells of the MBs may be required to maintain cellular homeostasis, allowing for olfactory learning.

The present findings suggest that holding a fine-tuned homeostatic balance in the cells of the MBs is critical for proper learning. In addition, signaling systems, such as the EGFR pathways that may not be directly involved in transmitting learning related content, may nevertheless be essential to enable learning.

\section{Materials and Methods}

\section{Fly strains and conditions}

Flies were cultured at $25^{\circ} \mathrm{C}$ on conventional corn meal, yeast, and glucose agar medium. All RNAi lines, UAS-spitz ${ }^{\text {RNAi }}$, UASkeren $^{\mathrm{RNAi}}$, and UAS-vein ${ }^{\mathrm{RNAi}}$ (Transformant ID 103817, 104299 and 109437, respectively) were obtained from the Vienna Drosophila RNAi Center (Austria). The UAS-EGFR ${ }^{\text {DN }}$ line (DGRC no. 108189) was from the Drosophila Genetics Resource Center (Japan). The UAS-secreted Spitz::GFP line was a gift from Benny Shilo (Rehovot, Israel). Elav-GeneSwitch containing a mifepristone-inducible elav-gal4 driver was kindly provided by Haig Keshishian (Yale University, USA), an n-synaptobrevin-gal4 (nsybgal4) line was a gift from Julie H. Simpson, (Janelia Farm, USA), and the driver line OK107-gal4 with predominant expression in the MBs was a gift from Dennis Pauls (University of Würzburg, Germany). The 20XUAS-IVS-mCD8::GFP line has the following genotype ( $\left.\mathrm{w}^{*} ; \mathrm{P}\{20 \mathrm{XUAS}-\mathrm{IVS}-\mathrm{mCD} 8:: \mathrm{GFP}\} \mathrm{attP} 2\right)$ and was selected because it shows no Gal4-independent expression in the brain. It was obtained from the Bloomington Stock Center (ID 32194). All other lines were from the Bloomington Drosophila Stock Center (USA).

\section{Generation of promoter-gal4 lines}

Presumptive promoter regions (fragments of $\sim 3000-4000 \mathrm{bp}$ located upstream of the translation start) of spitz (Dmel/spi, CG10334), vein (Dmel/vn, CG10491), keren (Dmel/Krn, CG 32179), and EGFR (Dmel/Egfr, CG10079) were amplified from genomic DNA using matching oligonucleotides (Biomers):

\section{pSpitz_fwd attB1CTCGTCTGGGTTTCATCCTG pSpitz_rev attB2GTAGCGTATCGGCTTGTTCG pVein_fwd attB1GATGATACAATGACGCTGCGC pVein_rev attB2CCAAAGTTGTTGCTGCTATTGC pKeren_fwd attB1CGTCAATTCGGCTTGAATTTGC pKeren_rev attB2GGAATTCTTGGCTTTGGCTG pEGFR_fwd attB1GTAACATCGAACCGGCTGAG pEGFR_rev attB2CGTTGTGGTGCCTAGTCAAG.}

The amplicons were cloned into pBPGUw (Addgene) using sitespecific recombination and subsequently injected into Drosophila embryos (Perrimon strain attP40) by BestGene Inc.

\section{Visualizing Gal4 expression patterns by immunofluorescence}

To visualize expression driven by the promoter-gal4 lines, these lines were crossed with the 20xUAS-IVS-mCD8::GFP line. Brains were dissected in $1 \times \mathrm{PBS}$, fixed in $4 \%$ paraformaldehyde/PBT ( $1 \times$ PBS, $0.3 \%$ Triton X-100) for $30 \mathrm{~min}$, washed with PBT, and, after blocking (5\% goat serum in PBT) for $30 \mathrm{~min}$, incubated with the primary antibody (rabbit anti-GFP [1:1000, Invitrogen] or mouse anti-nc82 [1:5, Developmental Studies Hybridoma Bank]) in blocking solution at $4^{\circ} \mathrm{C}$ overnight. After washing with PBT, brains were incubated with the secondary antibody (DyLight488-conjugated goat anti-rabbit or DyLight549-conjugated goat anti-mouse [both 1:400, Jackson ImmunoResearch Europe Ltd.]) in blocking solution at $4^{\circ} \mathrm{C}$ overnight in the dark. The samples were washed, and embedded in FocusClear (CelExplorer Labs Co.). Fluorescence microscopy was performed with the Axioplot Imager Z1 and Axiocam mRM camera (Carl Zeiss). For Z-projections, the Zeiss ApoTome was used.

\section{GeneSwitch experiments}

For experiments with inducible expression of transgenes and RNAi constructs, the elav-GeneSwitch driver line was crossed with the respective UAS-line. A stock solution of $250 \mu \mathrm{g} / \mathrm{mL} \mathrm{mi-}$ fepristone (Sigma-Aldrich) in 50\% ethanol was added during fly food preparation to a final concentration of $10 \mu \mathrm{g} / \mathrm{mL}$. For induction, $\mathrm{F}_{1}$ generation second-instar larvae were transferred to food 
medium supplemented with mifepristone. Larvae placed on food medium without mifepristone but containing the appropriate amount of $2 \%$ ethanol served as controls. The olfactory learning experiments were performed with third instar larvae $24 \mathrm{~h}$ $( \pm 1 \mathrm{~h})$ after the transfer. For RNAi experiments, eggs were laid on apple juice agar plates overnight and subsequently transferred to mifepristone containing medium or control medium.

\section{Olfactory learning experiments}

Olfactory learning experiments were performed at room temperature $\left(21-23^{\circ} \mathrm{C}\right)$ according to the method of Scherer et al. (2003), using the odors 1-octanol ([OCT], undiluted, Fluka) and amyl acetate ([AM], diluted 1:50 in paraffin oil, SAFC Supply Solutions) as well as the gustatory reinforcers fructose $([\mathrm{FRU},+], 2 \mathrm{M}$, Roth) as reward and quinine hemisulfate ([QUI, - ], 0.2\%, Sigma-Aldrich) as punishment. Lids of 200- $\mu$ L PCR tubes (Sarstedt) were utilized for odor presentation.

For the individual-animal assay, a group of eight larvae were trained together but tested individually, whereas for the en mass assay (Neuser et al. 2005) 12-17 larvae were trained and tested together. During the training, an inter-trial phase (Hendel et al. 2005) was inserted between the odor/taste presentations, in which the larvae remained on an empty agarose plate without odors or reinforcers for $1 \mathrm{~min}$. The training cycle was repeated four times (Fig. 2C). During the test phase, the position of the larvae was recorded every $30 \mathrm{sec}$ for a period of $5 \mathrm{~min}$ in the individual-animal assay and after $3 \mathrm{~min}$ in the en mass assay as "AM," "OCT," or "neutral" (a 5-mm-wide zone along the middle of the assay plate). The following formulas were used to estimate learning ability:

$$
\begin{aligned}
& \text { PREF } \mathrm{AM}_{\mathrm{AM}+/ \mathrm{OCT}-} \\
& =\frac{\text { (number of observations } \left.\mathrm{AM}_{\mathrm{AM}}-\text { number of observations } \mathrm{OCT}\right)}{\text { number of observations } \mathrm{s}_{\text {total }}} .
\end{aligned}
$$$$
\text { PREF } \mathrm{AM}_{\mathrm{AM}-/ \mathrm{OCT}+}
$$

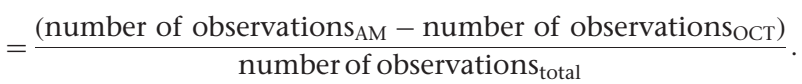

Subsequently, the learning index was calculated using PREF $\mathrm{AM}_{\mathrm{AM}+/ \mathrm{OCT}-}$ and PREF $\mathrm{AM}_{\mathrm{AM}-/ \mathrm{OCT}+}$ :

$$
\text { Learning index }=\frac{\left(\text { PREF AM } \mathrm{AM}+/ \mathrm{OCT}-_{-}-\mathrm{PREF} \mathrm{AM}_{\mathrm{AM}-/ \mathrm{OCT}+}\right)}{2} .
$$

\section{Gustatory test}

To test the ability of flies to taste FRU and QUI, a gustatory choice assay was performed according to the method of Hendel et al. (2005). In this assay, experimentally naive larvae can choose between the putative attractive taste of FRU and pure agarose or the putative repellent taste of QUI and pure agarose. Petri dishes (92 mm, Sarstedt) were filled with 1\% pure agarose (UltraPure, Invitrogen) on one side and either 2 -M FRU or $0.2 \%$ QUI in $1 \%$ agarose on the other side. Ten larvae were placed along the border of the tasting agent and pure agarose. After $15 \mathrm{~min}$, larvae were recorded as "FRU," "QUI," "pure" (without taste), or "neutral" (a 5$\mathrm{mm}$-wide zone along the middle of the assay plate or moved onto the lid). The preference for FRU or QUI was calculated by subtracting the number of larvae on the "pure" side from the number of larvae on the "taste" side and dividing the difference by the total number of larvae on the plate.

\section{Odor sensing}

To analyze the odor sensing ability of larvae, the to-be-associated odors AM or OCT were situated on one side of a Petri dish filled with $1 \%$ agarose and were tested versus an empty container. One experimentally naive larva was placed in the middle of the Petri dish and was allowed to move freely for $5 \mathrm{~min}$. The position of the larva was noted every $30 \mathrm{sec}$ as "AM," "OCT," "empty," or "neutral." Preference for the odor side was calculated by subtracting the number of counts on the empty side from the number of counts on the odor side and dividing the difference by the total number of counts.

\section{Statistical analysis}

The learning index values are represented by box plots (with medians as middle lines, $25 \%$ and $75 \%$ quantiles as box boundaries, and minimum and maximum values as whiskers). Each box plot of a learning experiment, with the exception of the en mass assay, displays a summary of the results of two biological replicates each performed in duplicate. Preferences for each replicate were sorted in ascending order to calculate the learning indices. Statistical analysis was performed using GraphPad Prism software version 3.0 (GraphPad Software). For comparisons between two groups, the Mann-Whitney test was used for nonparametric data. All learning indices were rounded to one decimal place.

\section{Acknowledgments}

We thank B.-Z. Shilo, H. Keshishian, and J.H. Simpson for providing flies. This work was supported by the Cluster of Excellence "Inflammation at Interfaces" of the German Research Council (DFG).

\section{References}

Aguirre A, Dupree JL, Mangin JM, Gallo V. 2007. A functional role for EGFR signaling in myelination and remyelination. Nat Neurosci 10: 990-1002.

Akalal DB, Yu D, Davis RL. 2010. A late-phase, long-term memory trace forms in the gamma neurons of Drosophila mushroom bodies after olfactory classical conditioning. J Neurosci 30: 16699-16708.

Arteaga CL. 2006. EGF receptor mutations in lung cancer: From humans to mice and maybe back to humans. Cancer cell 9: 421-423.

Bergmann A, Tugentman M, Shilo BZ, Steller H. 2002. Regulation of cell number by MAPK-dependent control of apoptosis: A mechanism for trophic survival signaling. Dev Cell 2: 159-170.

Blum AL, Li W, Cressy M, Dubnau J. 2009. Short- and long-term memory in Drosophila require cAMP signaling in distinct neuron types. Curr Biol 19: $1341-1350$.

Bonner JM, Boulianne GL. 2011. Drosophila as a model to study age-related neurodegenerative disorders: Alzheimer's disease. Exp Gerontol 46: $335-339$.

Brinkmann BG, Agarwal A, Sereda MW, Garratt AN, Muller T, Wende H, Stassart RM, Nawaz S, Humml C, Velanac V, et al. 2008. Neuregulin-1/ ErbB signaling serves distinct functions in myelination of the peripheral and central nervous system. Neuron 59: 581-595.

Busto GU, Cervantes-Sandoval I, Davis RL. 2010. Olfactory learning in Drosophila. Physiology 25: 338-346.

Chen-Plotkin AS, Hu WT, Siderowf A, Weintraub D, Goldmann Gross R, Hurtig HI, Xie SX, Arnold SE, Grossman M, Clark CM, et al. 2011. Plasma epidermal growth factor levels predict cognitive decline in Parkinson disease. Ann Neurol 69: 655-663.

Corl AB, Berger KH, Ophir-Shohat G, Gesch J, Simms JA, Bartlett SE, Heberlein U. 2009. Happyhour, a Ste20 family kinase, implicates EGFR signaling in ethanol-induced behaviors. Cell 137: 949-960.

Davis RL. 2004. Olfactory learning. Neuron 44: 31-48.

Drain P, Folkers E, Quinn WG. 1991. cAMP-dependent protein kinase and the disruption of learning in transgenic flies. Neuron 6: 71-82.

Fleck D, Garratt AN, Haass C, Willem M. 2011. BACE1 dependent neuregulin proteolysis. Curr Alzheimer Res 9: 178-183.

Foltenyi K, Greenspan RJ, Newport JW. 2007. Activation of EGFR and ERK by rhomboid signaling regulates the consolidation and maintenance of sleep in Drosophila. Nat Neurosci 10: 1160-1167.

Fraguas S, Barberan S, Cebria F. 2011. EGFR signaling regulates cell proliferation, differentiation and morphogenesis during planarian regeneration and homeostasis. Dev Biol 354: 87-101.

Gervasi N, Tchenio P, Preat T. 2010. PKA dynamics in a Drosophila learning center: Coincidence detection by rutabaga adenylyl cyclase and spatial regulation by dunce phosphodiesterase. Neuron 65: 516-529.

Hambardzumyan D, Parada LF, Holland EC, Charest A. 2011. Genetic modeling of gliomas in mice: New tools to tackle old problems. Glia 59: $1155-1168$.

Hannan F, Ho I, Tong JJ, Zhu Y, Nurnberg P, Zhong Y. 2006. Effect of neurofibromatosis type I mutations on a novel pathway for adenylyl 
cyclase activation requiring neurofibromin and Ras. Hum Mol Genet 15: 1087-1098.

Harris TJ, McCormick F. 2010. The molecular pathology of cancer. Nat Rev Clin Oncol 7: 251-265.

Harrison PJ, Law AJ. 2006. Neuregulin 1 and schizophrenia: Genetics, gene expression, and neurobiology. Biol Psychiatry 60: 132-140.

Hatanpaa KJ, Burma S, Zhao D, Habib AA. 2010. Epidermal growth factor receptor in glioma: Signal transduction, neuropathology, imaging, and radioresistance. Neoplasia 12: 675-684.

Hendel T, Michels B, Neuser K, Schipanski A, Kaun K, Sokolowski MB, Marohn F, Michel R, Heisenberg M, Gerber B. 2005. The carrot, not the stick: Appetitive rather than aversive gustatory stimuli support associative olfactory learning in individually assayed Drosophila larvae. J Comp Physiol A Neuroethol Sens Neural Behav Physiol 191: $265-279$.

Hidalgo A, Kinrade EF, Georgiou M. 2001. The Drosophila neuregulin vein maintains glial survival during axon guidance in the CNS. Dev Cell 1: 679-690.

Hochstrasser T, Ehrlich D, Marksteiner J, Sperner-Unterweger B, Humpel C. 2012. Matrix metalloproteinase-2 and epidermal growth factor are decreased in platelets of Alzheimer patients. Curr Alzheimer Res 9: 982-989.

Kojic N, Chung E, Kho AT, Park JA, Huang A, So PT, Tschumperlin DJ. 2010. An EGFR autocrine loop encodes a slow-reacting but dominant mode of mechanotransduction in a polarized epithelium. FASEB J 24: $1604-1615$.

Luo X, Prior M, He W, Hu X, Tang X, Shen W, Yadav S, Kiryu-Seo S, Miller R, Trapp BD, et al. 2011. Cleavage of neuregulin-1 by BACE1 or ADAM10 protein produces differential effects on myelination. J Biol Chem 286: 23967-23974.

Mimeault M, Batra SK. 2011. Animal models relevant to human prostate carcinogenesis underlining the critical implication of prostatic stem/ progenitor cells. Biochim Biophys Acta 1816: 25-37.

Moasser MM. 2007. The oncogene HER2: Its signaling and transforming functions and its role in human cancer pathogenesis. Oncogene 26: 6469-6487.

Neuser K, Husse J, Stock P, Gerber B. 2005. Appetitive olfactory learning in larvae: Effects of repetition, reward strength, age, gender, assay type and memory span. Anim Behav 69: 891-898.
Osterwalder T, Yoon KS, White BH, Keshishian H. 2001. A conditional tissue-specific transgene expression system using inducible GAL4. Proc Natl Acad Sci 98: 12596-12601.

Pauls D, Selcho M, Gendre N, Stocker RF, Thum AS. 2010. Drosophila larvae establish appetitive olfactory memories via mushroom body neurons of embryonic origin. J Neurosci 30: 10655-10666.

Pavlov IP. 1927. Conditioned reflexes: An investigation of the physiological activity of the cerebral cortex. Oxford University Press, London.

Read RD. 2011. Drosophila melanogaster as a model system for human brain cancers. Glia 59: 1364-1376.

Sato K, Touhara K. 2009. Insect olfaction: Receptors, signal transduction, and behavior. Results Probl Cell Differ 47: 121-138.

Scherer S, Stocker RF, Gerber B. 2003. Olfactory learning in individually assayed Drosophila larvae. Learn Mem 10: 217-225.

Selcho M, Pauls D, Han KA, Stocker RF, Thum AS. 2009. The role of dopamine in Drosophila larval classical olfactory conditioning. PloS One 4: e5897.

Shilo BZ. 2003. Signaling by the Drosophila epidermal growth factor receptor pathway during development. Exp Cell Res 284: 140-149.

Sibilia M, Kroismayr R, Lichtenberger BM, Natarajan A, Hecking M, Holcmann M. 2007. The epidermal growth factor receptor: From development to tumorigenesis. Differentiation 75: 770-787.

Stein RA, Staros JV. 2006. Insights into the evolution of the ErbB receptor family and their ligands from sequence analysis. BMC Evol Biol 6: 79.

Tang P, Steck PA, Yung WK. 1997. The autocrine loop of TGF-alpha/EGFR and brain tumors. J Neurooncol 35: 303-314.

Tomchik SM, Davis RL. 2009. Dynamics of learning-related cAMP signaling and stimulus integration in the Drosophila olfactory pathway. Neuron 64: $510-521$.

Venken KJ, Simpson JH, Bellen HJ. 2011. Genetic manipulation of genes and cells in the nervous system of the fruit fly. Neuron 72: 202-230.

Whitworth AJ. 2011. Drosophila models of Parkinson's disease. Adv Genet 73: $1-50$.

Woo RS, Lee JH, Yu HN, Song DY, Baik TK. 2011. Expression of ErbB4 in the neurons of Alzheimer's disease brain and APP/PS1 mice, a model of Alzheimer's disease. Anat Cell Biol 44: 116-127.

Received December 5, 2012; accepted in revised form January 10, 2013. 


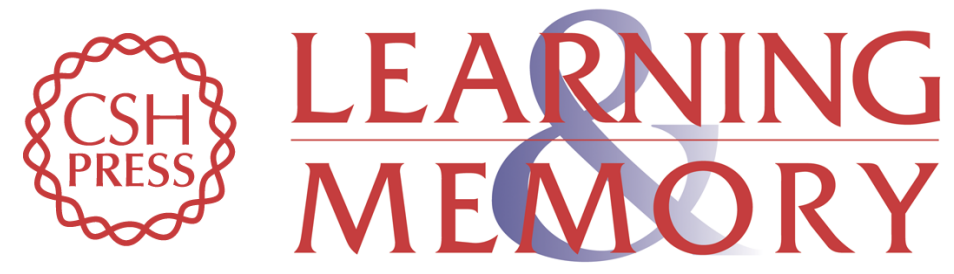

\section{EGFR signaling in the brain is necessary for olfactory learning in Drosophila larvae}

Tasja Rahn, Matthias Leippe, Thomas Roeder, et al.

Learn. Mem. 2013, 20:

Access the most recent version at doi:10.1101/Im.029934.112

Supplemental http://learnmem.cshlp.org/content/suppl/2013/03/18/20.4.194.DC1
Material

References This article cites 43 articles, 5 of which can be accessed free at: http://learnmem.cshlp.org/content/20/4/194.full.html\#ref-list-1

License

Email Alerting Receive free email alerts when new articles cite this article - sign up in the box at the Service top right corner of the article or click here. 